\title{
Interview with Loren D Walensky
}

Loren D Walensky is an Associate Professor of Pediatrics at Harvard Medical School, faculty member in the Department of Pediatric Hematology/Oncology at Dana-Farber Cancer Institute/Children's Hospital Boston, and Medical Director of Dana-Farber's Program in Cancer Chemical Biology. He graduated as valedictorian from Princeton University in 1990, majoring in Chemistry and receiving a Certificate in Science Policy from the Woodrow Wilson School of Public and International Affairs. He went on to receive his MD and PhD from the Johns Hopkins University School of Medicine in 1997. Dr Walensky spoke to Isaac Bruce, Commissioning Editor of Future Medicinal Chemistry, about his career in therapeutic peptides, his involvement in collaborations and funding, and the future of peptides as therapy.

At what stage in your career did you first become involved in peptide therapeutics and what first attracted your attention to this area?

I first became interested in peptide therapeutics as a pediatric hematology/oncology research fellow in the laboratory of Stanley Korsmeyer at the Dana-Farber Cancer Institute. In studying the apoptotic pathway, and BCL-2 family protein interactions in particular, we were interested in determining just how discretely structured peptide motifs embedded within the proteins selectively engaged their targets to impact the critical decision of life-or-death for the cell. Many BCL-2 proteins comprise a 3D cluster of $\alpha$-helices and if we could simulate these key bioactive domains in the laboratory, we felt that we could decode and potentially manipulate the interaction network.

At the time, Christian Schafmeister and Gregory Verdine at Harvard University had advanced a technique to generate chemically reinforced $\alpha$-helices by inserting non-natural olefinic amino acids into peptide sequences, followed by ruthenium-catalyzed olefin metathesis to form an all-hydrocarbon crosslink or 'staple'. With a background in synthetic organic chemistry from my undergraduate days in the laboratory of Ted Taylor at Princeton University and a PhD in molecular pharmacology from the laboratory of Solomon Snyder at Johns Hopkins University School of Medicine, I found this opportunity to blend the disciplines of chemistry and biology by bridging the laboratories of Korsmeyer and Verdine a perfect match for my postdoctoral research training.

2 How has having a medical background influenced your subsequent research?

As physicians and scientists, the projects we design and execute in the laboratory are driven by the palpable urgency to confront relapsed and refractory cancer; the clinical and research goals are inextricably linked. Caring for pediatric cancer patients has certainly changed my life - when you witness what your patients have to confront, you quickly learn not to sweat the small stuff in life. We fight alongside these remarkable children, doing everything we can to prevent cancer from robbing them of their futures. These children and their families are a constant source of inspiration to us - they compel us to think outside of the box and work that much harder. At the end of the day, we must do better for our patients because we still do not have the arsenal of cures that is so desperately needed.

Please can you update us on some of the research activities in which your group is currently engaged in relation to peptide therapeutics?

Having worked for more than 10 years on developing and applying stapled peptides as research tools and prototype therapeutics, time and again I have seen projects yield fundamentally new biological insights. That is, the use of a bioactive $\alpha$-helical peptide to probe a signaling pathway of interest has consistently revealed a new and previously unappreciated protein target, binding mode or biochemical mechanism. If stapled peptides can access these new interaction sites for biological discovery, to me that means they are uniquely poised to engage these complex protein interfaces for therapeutic benefit. To that end, we have structurally defined the elusive activation

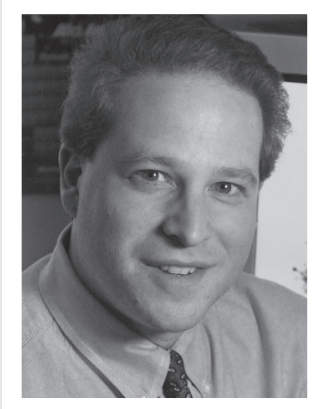

Loren D Walensky

Harvard Medical School, Dana-Farber Cancer Institute \& Children's Hospital Boston, Mayer Building, Room 664, 450 Brookline Avenue, Boston, MA 02215, USA

E-mail: loren_walensky@ dfci.harvard.edu 


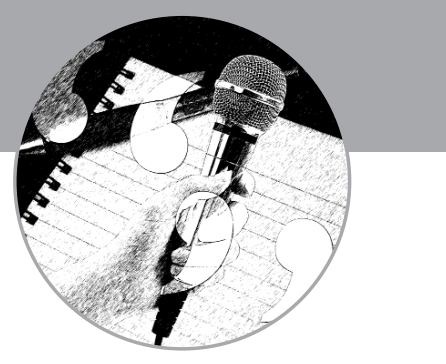

site on an essential executioner protein of the cell death pathway, dissected the key conformational changes that transform an inactive death protein into a toxic mitochondrial oligomer, identified a natural $\alpha$-helical peptide that can function as an exclusive inhibitor of a formidable anti-apoptotic protein linked to cancer, remedied the proteolytic instability of a lengthy peptide therapeutic, uncovered an unanticipated function for a death protein in metabolism and recapitulated the essential features of a key transcription factor motif to restore the tumor suppressor pathway in cancer cells.

Q At Harvard you are involved with the Dana-Farber Cancer Institute and the Children's Hospital Boston, among others; how does this collaborative effort affect your own research into novel peptide-based drugs?

Boston boasts a powerhouse of premier hospitals and research institutions, which are bustling with expertise spanning an ocean of scientific disciplines - collaborations abound. We have taken our stapled peptide projects to others in order to learn new techniques and expand our repertoire of analyses, and conversely, we are generating panels of stapled peptides for scientists interested in exploring their own applications and hypotheses. As a result, our collaborative projects cross many clinical and research fields, from cancer to diabetes and from proteomics to imaging.

You are on the Scientific Advisory Board of Aileron Therapeutics, which was founded due to the development of stapled peptides by yourself, Verdine and Korsmeyer at Harvard. Were you surprised by how quickly the field took off and the impact your work had?

There is no question that it has been a remarkable experience to see the postdoctoral research transform into a potential discovery engine for basic science and experimental therapeutics. The promise of structured peptide research is that evolutionarily honed protein domains, naturally optimized for potency and specificity, can themselves be tomorrow's drugs. The peptide therapeutics field is experiencing a revival because the previous obstacles of structural loss, peptide lability and cell impenetrability are being overcome.

You are supported by government-funded agencies as well as private organizations. How important is funding from the likes of The Burroughs Wellcome Fund, Stand Up to Cancer, the Leukemia and Lymphoma Society, the William Lawrence and Blanche Hughes Foundation, the Todd J Schwartz Fund and The Wolpoff Family Foundation in accelerating drug discovery for cancer?

In order to confront the biomedical challenges, we need an army of supremely talented trainees, and the instrumentation and scientific supplies to execute the research. This can only be accomplished by robust federal and private organization support. The more resources deployed, the more productive, efficient and impactful we can be. Each source of support fulfills a critical need. Federal grants fund comprehensive, long-term research goals that fundamentally enable our laboratory to exist. Support from societies and private organizations can fuel the critical early experiments of those high-risk 'fresh idea' projects that can ultimately be transformative. For example, our private funding has enabled us to expand our impact across diverse disciplines, purchase new instrumentation that has doubled and tripled our output, and allowed supremely talented young trainees to plant and nurture their own intellectual seeds. Simply put, the level of funding dictates the breadth and depth of our work.

When comparing small-molecule therapeutics and peptide-based drugs, one of the associated problems with peptides is that they are often difficult to deliver to the active site or that they have limited ADME properties. How has this changed in recent years and what research has provided you with greater hope for this being resolved?

The greatest promise of stapled peptides, aside from their unique capacity to target protein interaction surfaces potently and selectively, is their remarkable proteolytic resistance. In my view, this will be the game changer for peptide therapeutics. We have seen the PK properties of singly and doubly stapled peptides consistently and dramatically outpace those of the corresponding unmodified peptides. A doubly stapled HIV1 fusion inhibitor peptide was so acid-protease resistant in vitro that we were motivated to conduct an oral gavage study, in which we detected fully intact 36-amino acid-long stapled peptide in serum within $30 \mathrm{~min}$ of oral administration. 
There has been a wealth of papers discussing the benefits of combining a diagnostic and therapeutic in a single agent: so-called 'theranostics', many of which are peptide-based. Do you believe that this strategy is beneficial over the two individual approaches?

An effective all-in-one approach that matches the sensitivity, specificity, safety and efficacy of individual approaches could be very beneficial to both patient and caregiver. Ideally, the agent used to identify the disease target for diagnostic purposes may ultimately be best suited to modulate the target. Indeed, stapled peptides are promising 'theranostic' candidates.

Q Are you concerned about the number of peptide-based therapies being approved to the market and do you feel the cost of peptide synthesis is having an impact on this?

In the context of the myriad unmet medical needs we face as patients and physicians, more is more and less is less. The number of approved peptide therapies will be driven by the clinical successes. The greatest concern I face is having a patient with relapsed cancer and nothing efficacious to prescribe.

Historically, the chemistry and cost of peptide synthesis was a barrier for developmental peptide therapeutics. However, with a variety of more efficient coupling chemistries and superior highthroughput automation, peptide synthesis costs have become more contained. As a result, there is less of an economic barrier, which will hopefully foster the expansion of peptide-based therapeutics.

What words of encouragement would you give young scientists who might be envisioning a career in peptide therapeutics?

Peptides such as insulin and other hormones that address extracellular targets have a long-standing history of success in a variety of disease areas. The development of structured peptides to engage extracellular and intracellular targets provides a new opportunity to expand the arsenal of therapeutic peptides and fulfill the promise of converting nature's protein-targeting domains into a new generation of therapies. Although much remains to be learned about the promise and potential of peptides as therapeutics, a career in this arena offers a unique opportunity to blend chemistry, biochemistry, cell biology and medicine in advancing a new generation of experimental therapies.

\section{Financial \& competing interests disclosure}

LD Walensky is a scientific advisory board member and consultant for Aileron Therapeutics. The author has no other relevant affiliations or financial involvement with any organization or entity with a financial interest in or financial conflict with the subject matter or materials discussed in the manuscript apart from those disclosed.

No writing assistance was utilized in the production of this manuscript. 